\title{
Loss of diacylglycerol kinase $\varepsilon$ causes thrombotic microangiopathy by impairing endothelial VEGFA signaling
}

\author{
Dingxiao Liu, ${ }^{1,2}$ Qiong Ding, ${ }^{1}$ Dao-Fu Dai, ${ }^{3}$ Biswajit Padhy, ${ }^{1}$ Manasa K. Nayak,, Can Li, ${ }^{1}$ Madison Purvis, \\ Heng Jin, ${ }^{4}$ Chang Shu, ${ }^{2}$ Anil K. Chauhan, ${ }^{1}$ Chou-Long Huang, ${ }^{1}$ and Massimo Attanasio ${ }^{1}$ \\ 'Department of Internal Medicine, University of lowa, lowa City, lowa, USA. ${ }^{2}$ Department of Vascular Surgery, The Second \\ Xiangya Hospital, Central South University, Changsha, China. ${ }^{3}$ Department of Pathology, University of lowa, lowa City, \\ lowa, USA. ${ }^{4}$ Department of Emergency Medicine, Tianjin Medical University General Hospital, Tianjin, China.
}

\begin{abstract}
Loss of function of the lipid kinase diacylglycerol kinase $\varepsilon$ (DCK\&), encoded by the gene DCKE, causes a form of atypical hemolytic uremic syndrome that is not related to abnormalities of the alternative pathway of the complement, by mechanisms that are not understood. By generating a potentially novel endothelial specific $D g k e$-knockout mouse, we demonstrate that loss of $D g k e$ in the endothelium results in impaired signaling downstream of VEGFR2 due to cellular shortage of phosphatidylinositol 4,5-biphosphate. Mechanistically, we found that, in the absence of DCKE in the endothelium, Akt fails to be activated upon VEGFR2 stimulation, resulting in defective induction of the enzyme cyclooxygenase 2 and production of prostaglandin $E_{2}\left(P G E_{2}\right)$. Treating the endothelial specific $D g k e$-knockout mice with a stable $\mathrm{PCE}_{2}$ analog was sufficient to reverse the clinical manifestations of thrombotic microangiopathy and proteinuria, possibly by suppressing the expression of matrix metalloproteinase 2 through PGE-dependent upregulation of the chemokine receptor CXCR4. Our study reveals a complex array of autocrine signaling events downstream of VEGFR2 that are mediated by PGE$_{2}$, that control endothelial activation and thrombogenic state, and that result in abnormalities of the glomerular filtration barrier.
\end{abstract}

Conflict of interest: The authors have declared that no conflict of interest exists.

Copyright: (c) 2021, Liu et al. This is an open access article published under the terms of the Creative Commons Attribution 4.0 International License.

Submitted: December 21, 2020

Accepted: March 25, 2021

Published: May 10, 2021

Reference information: /CI Insight. 2021;6(9):e146959.

https://doi.org/10.1172/jici. insight.146959.

\section{Introduction}

Atypical hemolytic uremic syndrome (aHUS) is a form of thrombotic microangiopathy (TMA) characterized by hemolytic anemia, thrombocytopenia, and acute renal failure not associated with enteric infections caused by Shiga toxin-producing bacteria. TMA in aHUS affects invariably the microvasculature in the renal glomeruli, causing renal insufficiency. Mutations in complement and complement-regulating genes have been identified in about $50 \%$ of idiopathic aHUS cases, pointing at the central role of the complement cascade in this disease (1). Notwithstanding, we and others described a genetic form of aHUS that is caused by mutations in the gene $D G K E$ that encodes the lipid kinase diacylglycerol kinase $\varepsilon(D G K \varepsilon)$, revealing that factors unrelated to the complement system can cause aHUS by mechanisms that are not understood $(2,3)$

$\mathrm{DGK} \varepsilon$ is a lipid kinase with high specificity for diacylglycerol (DAG) conjugated with arachidonic acid (AA) at the second carbonyl group of DAG (4-7). As an effect of the DGKe activity, cellular arachidonoyl poly-phosphatidyl-inositides (PtdIns) predominate over PtdIns acylated with other acyl moieties, and, as a consequence, in Dgke-knockout cells the total amount of PtdIns is 3-fold or lower than in WT cells (4-8). Under basal conditions phosphatidyl-inositol diphosphate $\left[\operatorname{PtdIns}(4,5) P_{2}\right.$, henceforth PIP2] is the most abundant cellular PtdIns. Importantly, it is consumed upon activation of multiple receptors, including receptor tyrosine kinases such as VEGFR2, and it needs to be regenerated continuously through the phosphoinositide cycle (Figure 1A) (9-12).

Here, based on in vitro studies on primary endothelial cells and on the use of an endothelial specific Dgke-knockout mouse, we show that the signaling downstream of VEGFR2 is compromised in the absence of DGKe in the endothelium because of impaired activation of Akt and that the latter is restored by increasing cellular levels of PIP2. Mechanistically, we found that defective Akt activation in endothelial cells results in lack of expression of the gene prostaglandin-endoperoxide synthase 2, which encodes the enzyme cyclooxygenase 2 ( $\mathrm{Cox} 2)$ and induces the synthesis of its main product prostaglandin $\mathrm{E}_{2}\left(\mathrm{PGE}_{2}\right)$. 
A

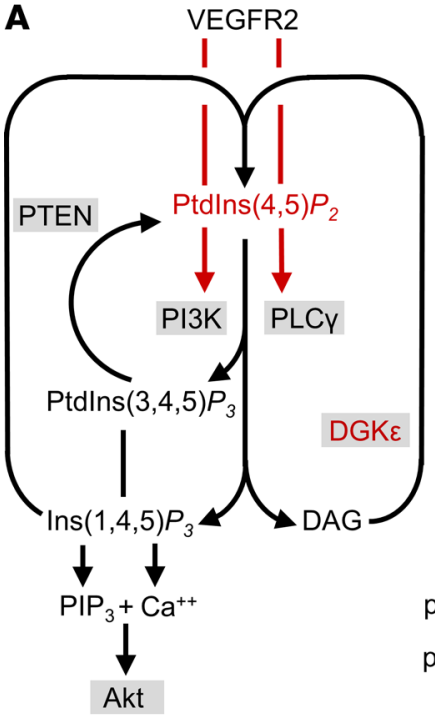

B

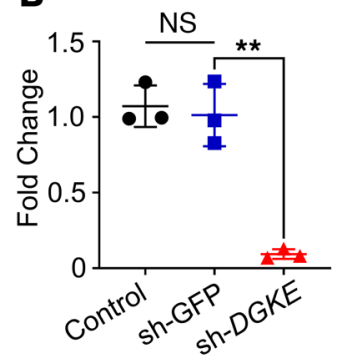

C

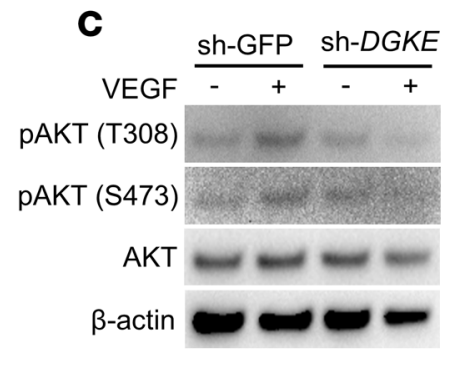

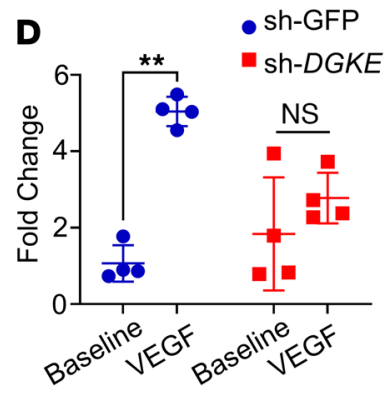

$\mathbf{F}$

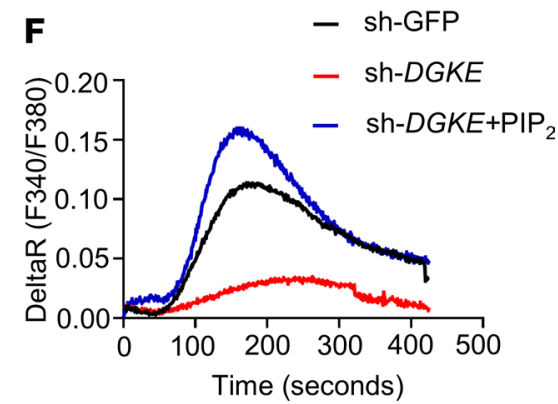

Figure 1. VEGFR2-dependent Akt activation is compromised in DGKE-knockdown human umbilical vein epithelial cells. (A) Schematic representation of the phosphoinositide cycle and of some of the enzymes involved in the cycle (boxes). Red arrows point to the major enzymes (PI3K and phospholipase $C$ $\gamma$, PLC $\gamma$ ) activated downstream of VEGFR2. Black arrows represent generation of Ptdlns $(1,4,5) P_{3}(P I P 3)$ and Ca ${ }^{2+}$ downstream of PI3K and PLC $\gamma$, respectively, and activation of Akt. The common substrate of PI3K and PLC $\gamma$, PIP2, required for Akt activation is highlighted in red. DCKE is highlighted in red. (B) Efficiency of the sh-RNA knockdown in HUVECs measured by quantitative PCR (Q-PCR) compared with nontargeted control (sh-GFP) cells. Control: nontransfected cells. (C) Western blot showing impaired Akt activation (phosphorylation of threonine 308 and serine 473 ) in the shRNA-knockdown HUVECs upon VEGFA stimulation, compared with nontargeted control cells. (D) Expression of Cox2, measured by Q-PCR, is not induced in DGKE-knockdown HUVECs compared with nontargeted control cells stimulated with VEGFA. (E) Western blots showing that impaired Akt activation in the sh-RNA knockdown HUVECs upon VEGFA stimulation is partially reversed by PIP2 and PIP3 supplementation. (F) Changes in intracellular Ca ${ }^{2+}$ concentrations after VEGFA supplementation in DGKE-knockdown HUVECs, in DGKE-knockdown HUVECs after PIP2 supplementation, and in nontargeted HUVEC controls over time, measured by Fura-2 AM fluorescence. Data are from 3-4 independent experiments and are presented as mean \pm SD. **: $P<0.01$ by 1 -way ANOVA in B and by Student's $t$ test in D. Each data point represents 1 experiment. PTEN, phosphatase and tensin homolog; pAKT, phosphorylated Akt; DeltaR: fluorescence ratio as $340 \mathrm{~nm} / 380 \mathrm{~nm}$.

Treating endothelial specific Dgke-knockout mice with a stable $\mathrm{PGE}_{2}$ analog reversed not only the aHUS, but also the proteinuria, possibly through CXCR4-mediated downregulation of matrix metalloproteinase 2 (MMP-2). Our data indicate that the endothelium is the tissue that is primarily affected by loss of $D g k e$ and reveal multiple complex autocrine signaling events downstream of VEGFR2 that result in endothelial activation, thrombogenic state, and abnormalities of the glomerular filtration barrier. These signaling steps may be manipulated to modify the clinical course of the DGKE-related aHUS and conceivably of other diseases caused by endothelial activation or abnormal angiogenesis.

\section{Results}

VEGFR2-dependent Akt activation is compromised in DGKE-knockdown human umbilical vein endothelial cells and in human microvascular endothelial cells. VEGFR2 is expressed in endothelial cells and exerts proproliferative, prosurvival, and promigratory functions, promoting angiogenesis upon stimulation by VEGFA (13-17). VEGFR2 signals downstream by autophosphorylating multiple tyrosine residues, where effector proteins are recruited (14). This results in, among other effects, activation of PLC $\gamma$, which then hydrolyzes PIP2 into inositol 1,4,5-triphosphate $\left[\operatorname{Ins}(1,4,5) P_{3}\right.$, hereafter IP3] and DAG, and activation of phosphatidylinositol-3-kinase (PI3K), which leads to the generation of PIP3 from its precursor, PIP2, and ultimately to the activation of Akt (Figure 1A) (18-22). Since VEGF signaling is crucial to the maintenance of the glomerular microvasculature $(23,24)$, and because the disruption of VEGF signaling in humans and mice results in glomerular lesions that resemble those observed in humans with loss-of-function mutations in $D G K E(2$, 25), we hypothesized that the loss of $D G K E$ may impair VEGF signaling in endothelial cells because of the shortage of PIP2 (Figure 1A). To test this hypothesis, we knocked down DGKE in human umbilical vein endothelial cells (HUVECs) by shRNA interference, using a nontargeting construct (sh-GFP) as a control 
(Figure 1B), and tested the activation of Akt by Western blot (phosphorylation of threonine 308 and serine 473 of Akt) upon VEGFA stimulation. Unlike untransfected and sh-GFP-transfected cells, DGKE-knockdown cells failed to activate Akt when exposed to VEGFA (Figure 1C and Supplemental Figure 1, A and B; supplemental material available online with this article; https://doi.org/10.1172/jci.insight.146959DS1). Consistent with the impaired Akt activation, the expression of Cox2, which in endothelial cells is induced by VEGFA (26-29), was also compromised (Figure 1D). However, Akt activation was partially rescued when cells were exposed to PIP2 and, as expected, to PIP3 (Figure 1E and Supplemental Figure 1, C and D). Likewise, intracellular calcium levels that are mobilized from the endoplasmic reticulum by increased intracellular concentrations of IP3 did not rise after VEGFA stimulation, but they did increase after supplementing the culture medium with PIP2 (Figure 1F). We obtained overlapping results using human microvascular endothelial cells (HMECs) (Supplemental Figure 2, A and B). These results indicate that Akt activation downstream of VEGFR2 is defective in DGKE-knockdown endothelial cells because of decreased availability of PIP2.

Endothelial specific Dgke-knockout mice fully recapitulate the human disease. To test if VEGFA signaling in endothelial cells lacking Dgke is also compromised in vivo, we generated a conditional knockout mouse in which exon 2 of $D g k e$ was deleted in a tissue-specific manner upon Cre recombination, using knockout first embryonic stem (ES) cells from the Knockout Mouse Project (KOMP) repository (30, 31). C57BL/6 Dgke-floxed mice are viable and fertile, and they produce progeny at the expected Mendelian ratio. We confirmed that exon 2 of $D g k e$ is excised upon Cre recombination, by using the CMV-Cre mice to delete $D g k e$ in all tissues (32) (Figure 2A). We also verified that the expression of the transgene is tissue specific, by generating ${ }^{\mathrm{Nphs} 2 \mathrm{Cre}} D g k e^{\mathrm{LacZ}}$ knockin mice and showing that the LacZ expression was present exclusively in the glomeruli $(30,33)$ (Figure 2B). To generate endothelial specific Dgke-knockout mice, $D g k e$-floxed mice were then crossed to mice in which the Cre recombinase is driven by the angiopoietin receptor Tie 2 promoter in endothelial cells and in $2 \%$ to $7 \%$ of blood mononuclear cells (34). We assessed the knockout efficiency by real-time PCR (RT-PCR) on RNA extracted from ${ }^{\text {Tie2Cre }} D g k e^{\mathrm{f} / \mathrm{fl}}$ lungs, which is one of the most vascularized tissues (Figure $2 \mathrm{C}$ ), given that no antibody is available that reliably detects Dgke. We excluded the possibility that abnormal platelet function due to expression of Tie2 in megakaryocytes could contribute to the observed phenotype by performing in vitro studies of platelet aggregation (Supplemental Figure 3). Unlike the constitutive Dgke-knockout mice that do not have spontaneous phenotype, endothelial specific ${ }^{\text {Tie2Cre }} D g k e^{\mathrm{f} / \mathrm{fl}}$ conditional knockout mice developed occlusion of the glomerular capillaries (Figure 2, D and E), schistocytosis (Figure 2, F and G, and Supplemental Figure 4A), hemolytic anemia (Figure 2H and Supplemental Figure 4B), thrombocytopenia (Figure 2I), and renal insufficiency as early as 2 months of age (Figure 2J). Remarkably, these mice also developed albuminuria (Figure $2 \mathrm{~K}$ ), which is observed in all patients affected by DGKE disease, by 3 months of age. The presence of albuminuria in endothelial specific ${ }^{\text {Tie2 } \mathrm{Cre}} D g k e^{\mathrm{fl} / \mathrm{fl}}$ knockout mice and its appearance at a later time, compared with the renal insufficiency, suggest that the endothelium is the cellular compartment responsible for the DGKE disease and that the impairment of the glomerular barrier may be a later and secondary event. Interestingly, we did not detect obvious signs of TMA in liver, small intestine, and spleen arterioles of ${ }^{\mathrm{Tie} 2 \mathrm{Cre}} D g k e^{\mathrm{fl} / \mathrm{fl}}$ knockout mice (Supplemental Figure 5), which may indicate a particular susceptibility of the glomerular microvasculature to this disease.

A phosphatase and tensin homolog inhibitor rescues the phenotype of endothelial specific Dgke-knockout mice. As observed in the DGKE-knockdown cells, Akt phosphorylation was lower in kidney cortexes of ${ }^{\text {Tie2Cre }} D g k e^{\mathrm{fl} /}$ ${ }^{\mathrm{fl}}$ mice, compared with controls (Figure 3A, lanes 1 through 6; and Supplemental Figure 1, E and F). Hence, we tested whether increasing the endothelial levels of PIP3 would rescue the phenotype in vivo. To this end, we crossed ${ }^{\mathrm{Tie} 2 \mathrm{Cre}} D g k e^{\mathrm{f} / \mathrm{fl}}$ mice to Pten-floxed mice (35), to obtain ${ }^{\mathrm{Tie} 2 \mathrm{Cre}} D g k e^{\mathrm{f} / \mathrm{fl}} P t e n^{+/ \mathrm{fl}}$ in which only 1 of the 2 Pten alleles is present in endothelial cells; however, we were not able to obtain pups of the desired genotype, likely because of in utero mortality. For this reason, we used a potent small molecule inhibitor of PTEN (VO-OHpic) (36), and injected 8-week-old ${ }^{\text {Tie2Cre }} D g k e^{\mathrm{A} / \mathrm{fl}}$ mice i.p. daily for 4 weeks. As expected, treating the ${ }^{\text {Tie2Cre }} D g k e^{\mathrm{f} / \mathrm{fl}}$ mice with the PTEN inhibitor resulted in increased Akt activation in renal cortexes (Figure 3A, lanes 4 through 9; and Supplemental Figure 1, E and F). Consistent with Akt activation, the expression of Cox 2 in kidney cortexes was increased in ${ }^{\text {Tie2Cre }} D g k e^{\mathrm{f} / \mathrm{fl}}$ mice treated with PTEN inhibitor compared with vehicle-treated ${ }^{\text {Tie2Cre }} D g k e^{\mathrm{f} / \mathrm{fl}}$ mice (Figure 3B and Supplemental Figure 1G). Remarkably, VO-OHpic-treated mice were protected from developing glomerular capillary obstruction (Figure 3, C and D), thrombocytopenia (Figure 3H), renal insufficiency, and proteinuria (Figure 3, I and J). 

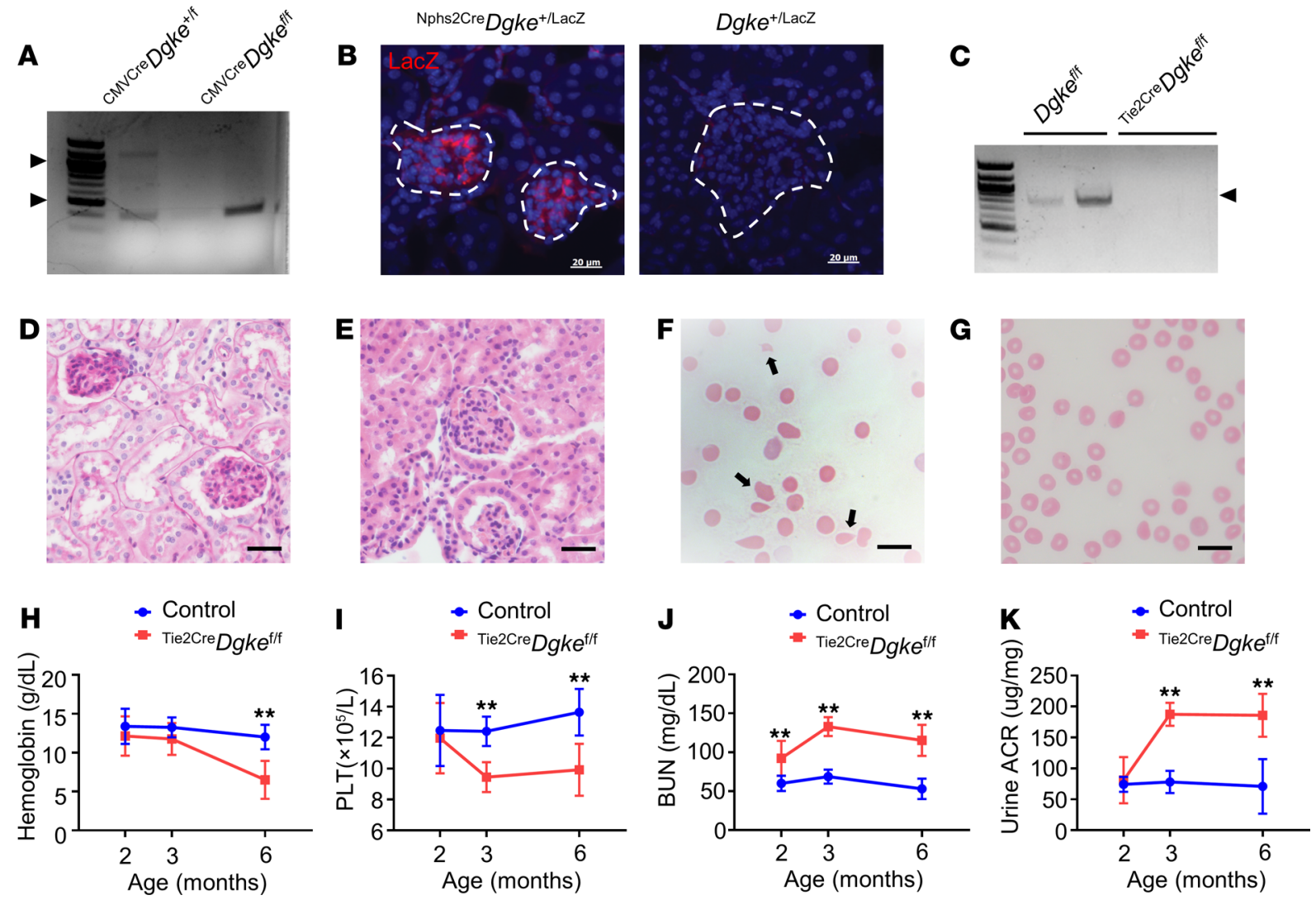

Figure 2. Endothelial specific $D g k e$-knockout mice recapitulate the full human phenotype. (A) Agarose gel of the PCR products of the amplification of genomic DNA from ubiquitously Cre-expressing ${ }^{\mathrm{CMVCre}} D g \mathrm{ke}^{\mathrm{fl} / \mathrm{fl}}$ mice compared with ${ }^{\mathrm{CMVCre}} \mathrm{Dg} \mathrm{ke}^{+/ f \mathrm{fl}}$ heterozygotes. Heterozygous mice show a long band of $1201 \mathrm{bp}$ corresponding to the retained exon 2 and a shorter band of 365 bp corresponding to the excised exon 2 (arrowheads). Exon 2 is not retained in the homozygous status. (B) Immunofluorescence microscopy images of glomeruli (dashed lines) from ${ }^{\text {Nphs2Cre }} D g k e^{+/ L a c z} \mathrm{knockin}$ mice compared with $D g k e^{+/ L a c z}$ controls, showing LacZ expression exclusively in glomeruli. Scale bars are $20 \mu \mathrm{m}$. (C) RT-PCR on RNA showing a band of 830 bp (arrowhead) in Dgkef/fl mice and no product in ${ }^{\text {Tiezcre }} D g k e^{f / f f l}$ mice. Primers were placed in the corresponding exon 1 and 5 in the cDNA of $D g k e$. (D) Representative bright-field microscopy images of glomeruli of Periodic acid-Schiff-stained (PAS-stained) and (E) H\&E-stained sections of ${ }^{\text {Tiezcre }} D g k e^{\text {fl/fl }}$ mouse kidneys showing near-complete occlusion of the capillary tuft. Scale bars are $50 \mu \mathrm{m}$. (F) Smears of blood from ${ }^{\text {Tiezcre }} D g k e^{\mathrm{fl} / \mathrm{fl}}$ and (C) $D g k e^{\mathrm{fl} / \mathrm{fl}}$ controls at 6 months of age. Numerous schistocytes (arrows) are present in ${ }^{\text {Tiezcre }} D g k e^{\mathrm{fl} / \mathrm{fl}}$ knockouts. Scale bars are $75 \mu \mathrm{m}$. (H-K) Serum hemoglobin, circulating platelets (PLT), blood urea nitrogen (BUN), and urine albumin to creatinine ratio (ACR) in ${ }^{\text {Tiezcre }} D g k e^{\mathrm{fl} / \mathrm{fl}}$ compared with $D g k e^{\mathrm{fl} / f l}$ controls at 2,3 , and 6 months of age. Data are presented as mean \pm SD. **: $P<0.01$ by Student's $t$ test. $n=6$ mice per group.

Although schistocytosis was clearly evident in peripheral blood smears of vehicle-treated mice (Figure 3, $\mathrm{E}$ and F, and Supplemental Figure 4C), the serum hemoglobin concentrations did not significantly differ from those of VO-OHpic-treated mice (Figure 3G). These results indicate that Akt activation is impaired in kidneys of endothelial specific knockout mice and that increasing PIP3 cellular levels is sufficient to rescue the symptoms in ${ }^{\text {Tie2Cre }} D g k e^{\mathrm{A} / \mathrm{l}}$ knockout mice.

Overexpression of Cox2 in endothelial cells rescues the phenotype of ${ }^{\text {Tie2Cre }} D g k e^{\mathrm{n} / \mathrm{fl}}$ mice. We previously reported that the expression of the gene prostaglandin-endoperoxide synthase 2, also known as cyclooxygenase 2 ( $\operatorname{Cox} 2)$, and the synthesis of its main product $\mathrm{PGE}_{2}$ were impaired in Dgke constitutive knockout mice (37). However, still undetermined is if impaired Cox2 induction is only associated with or determines the phenotype in $D g k e$-knockout mice. To answer this question, we crossed ${ }_{\text {Tie2Cre }} D g k e^{\mathrm{f} / \mathrm{fl}}$ mice with B6.129S4-Tg(CAG-EGFP,-Ptgs2,-hrluc)(Cox2-COE) mice to obtain ${ }^{\text {Tie2Cre }} D g k e^{\mathrm{f} / / \mathrm{fl}}$ Cox2-COE double transgenics. Cox2-COE mice were engineered to carry a transgene that contains a CAG promoter that drives the expression of Cox2. The promoter is followed by a loxP-flanked sequence containing enhanced GFP and a transcriptional/translational STOP sequence that allows tissue-specific overexpression of Cox2 in the presence of Cre recombinase (38). As observed in ${ }^{\text {Tie2Cre }} D g k e^{\mathrm{f} / \mathrm{fl}}$ mice treated with the PTEN inhibitor, ${ }^{\mathrm{Tie} 2 \mathrm{Cre}} D g k e^{\mathrm{f} / / \mathrm{fl}}$ Cox2-COE mice did not develop glomerular capillary occlusion (Figure 4, A and B), schistocytosis and hemolytic anemia (Figure 4, C-E, and Supplemental Figure 4D), thrombocytopenia (Figure 4F), and renal insufficiency and proteinuria 
A

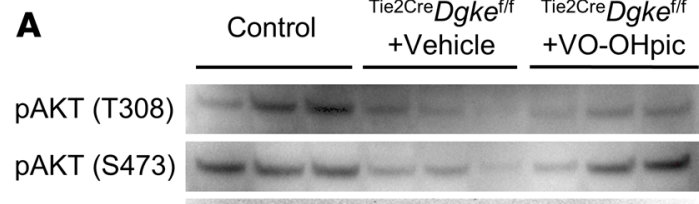

AKT

$\beta$-actin

C
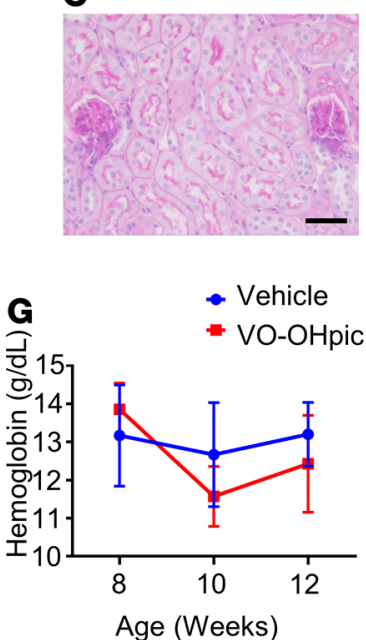

D

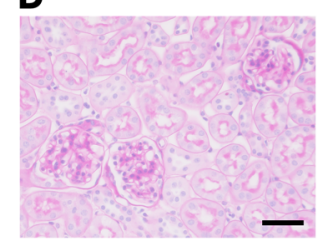

H

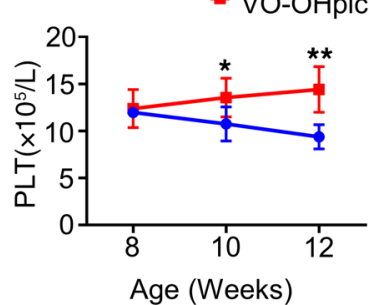

B

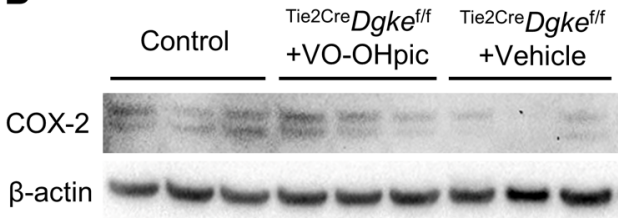

E

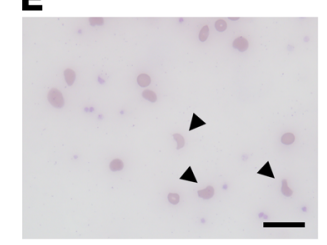

$\mathbf{F}$
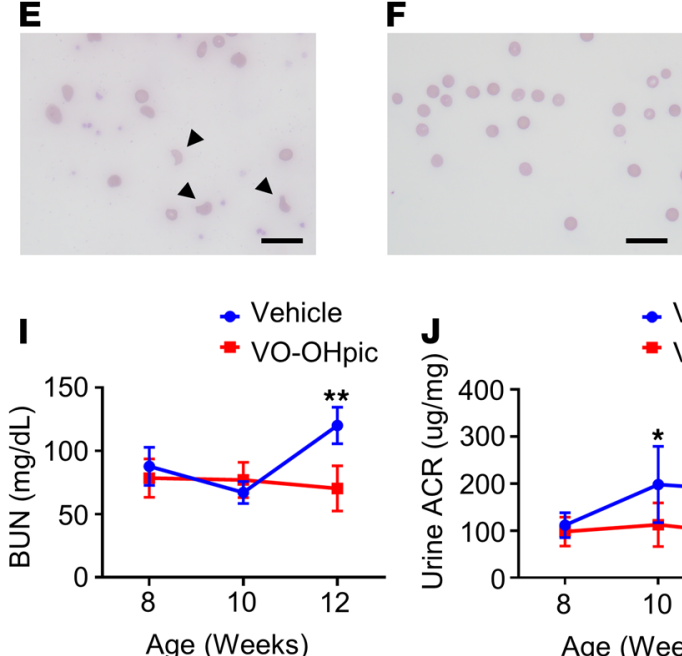

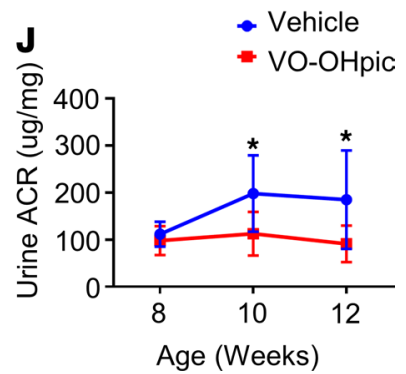

Figure 3. A PTEN inhibitor rescues the phenotype of endothelial-specific Dgke-knockout mice. (A) Western blot showing impaired Akt activation (phosphorylation of threonine 308 and serine 473 ) in kidney cortex extracts of ${ }^{\text {Tiezcre }} \mathrm{Dgk} \mathrm{e}^{\mathrm{fl} / \mathrm{fl}}$ mice compared with controls. Akt activation was partially rescued

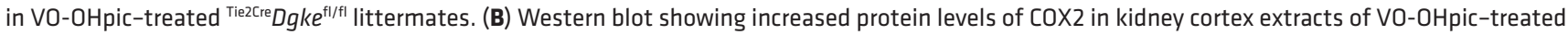
${ }^{T i e 2 C r e} D g k e^{f / f l}$ mice compared with ${ }^{T i e 2 C r e} D g k e^{f / f f l}$ littermates. (C) Representative bright-field microscopy images of glomeruli of PAS-stained Tiezcre $D g k e^{f l / f l}$ mouse kidneys and (D) ${ }^{\text {Tiezcre }} D g k e^{\text {fl/fl }}$ littermates' kidneys after 4 weeks of treatment with the PTEN inhibitor VO-OHpic. The occlusion of the glomerular

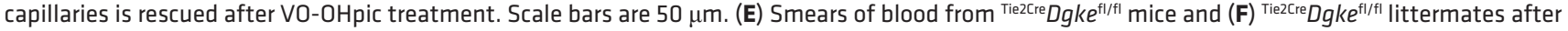
VO-OHpic treatment at 3 months of age. Schistocytes are found in Tie2cre Dgke ${ }^{\mathrm{fl} / \mathrm{fl}}$ mice (arrowheads) but not in VO-OHpic-treated mice. Scale bars are 50 $\mu \mathrm{m}$. (G-J) Serum hemoglobin, circulating PLT, BUN, and urine ACR in TiezCre Dgke ${ }^{\mathrm{fl} / \mathrm{fl}}$ mice compared with V0-OHpic-treated ${ }^{\text {TiezCre }} \mathrm{Dgke} \mathrm{fl}^{\mathrm{fl} / \mathrm{fl}}$ littermates at 8,10 , and 12 weeks of age. Data are presented as mean \pm SD. ${ }^{*}: P<0.05,{ }^{*}: P<0.01$ by Student's $t$ test. $n=6$ mice per group.

(Figure 4, G and H). These results show that the lack of endothelial expression of Cox2 is causative of the Dgke phenotype. They also denote that the failed induction of Cox 2 in endothelial cells and not the lack of its substrate AA causes aHUS and proteinuria in ${ }^{\text {Tie2Cre }} D g k e^{\text {fi/fl }}$ mice.

A stable PGE $E_{2}$ analog rescues the phenotype of endothelial specific Dgke-knockout mice. Cox2 is the rate-limiting enzyme for the generation of $\mathrm{PGE}_{2}$, a potent promoter of endothelial cell migration, survival, and angiogenesis $(39,40)$. VEGFA induces Cox2 and $\mathrm{PGE}_{2}$ in endothelial cells $(26,28,29,41)$, which suggests that its proangiogenic effect might be mediated at least partly by the production of $\mathrm{PGE}_{2}$ by mechanisms that are not completely understood $(27,42,43)$. In a precedent work we showed that urinary concentration of $\mathrm{PGE}_{2}$, but not of the stable $\mathrm{PGI}_{2}$ metabolite 6-keto $\mathrm{PGF}_{1 \mathrm{a}}$, was reduced in the Dgke constitutive knockouts compared with control mice. Dgke-knockout mice showed defective vascularization of surgical sponges implanted subcutaneously, and this defect was rescued by injecting the sponges with $\mathrm{PGE}_{2}$ (44), which pointed to the existence of a defect of $\mathrm{PGE}_{2}$-mediated angiogenesis in these mice. To clarify the role of $\mathrm{PGE}_{2}$ in determining the clinical manifestations in ${ }^{\text {Tie2Cre }} D g k e^{\mathrm{A} / \mathrm{fl}}$ mice, we tested if increasing circulating levels of $\mathrm{PGE}_{2}$ would rescue the hematologic and renal manifestations in our mouse model. To this end, we implanted subcutaneous osmotic pumps in 8-week-old ${ }^{\text {Tie2Cre }} D g k e^{\mathrm{A} / \mathrm{fl}}$ mice to continuously deliver the stable $\mathrm{PGE}_{2}$ analog 16,16 dimethyl-PGE ${ }_{2}\left(\mathrm{dmPGE}_{2}\right)$ and looked for changes in the phenotype after 4 weeks. Infusion of $\mathrm{dmPGE}_{2}$ was sufficient to normalize all the clinical manifestations in ${ }^{\text {TieCre }} D g k e^{\mathrm{A} / \mathrm{fl}}$ mice (Figure 5, A-H, and Supplemental Figure 4E). These results indicate that impaired $\mathrm{PGE}_{2}$ production is a key factor in determining the presence of disease in the absence of Dgke and that the anemia observed in these mice is hemolytic and not caused by bone marrow failure.

Treatment with a PGE $E_{2}$ stable analog normalizes endothelial cell activation and prothrombotic diathesis in ${ }^{\text {Tie2Cre }} D g k e^{\mathrm{A} / \mathrm{ll}}$ mice. $D G K E$-knockdown endothelial cells have been shown to express markers of activation 
A
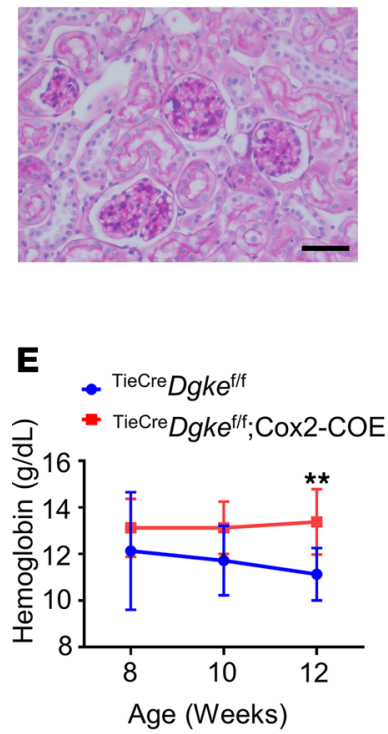

B

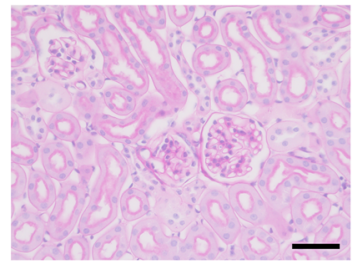

$\mathbf{F}$

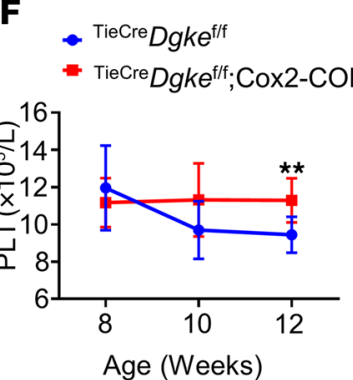

C

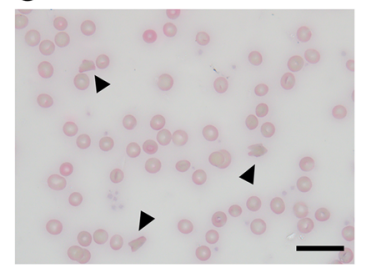

G

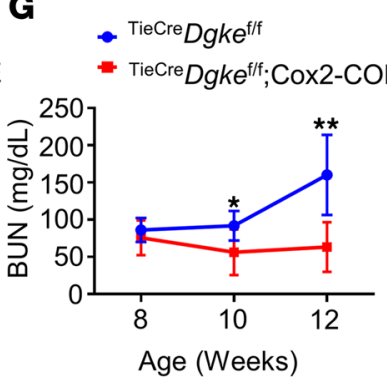

D
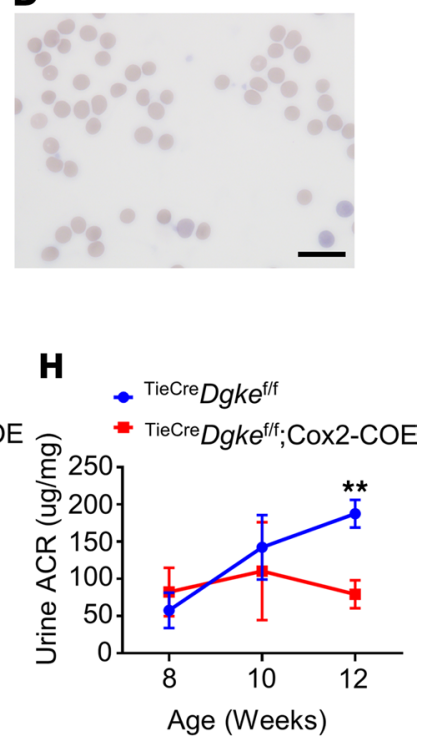

Figure 4. Overexpression of Cox2 in endothelial cells rescues the phenotype of endothelial specific $\mathbf{D g k e}$-knockout mice. (A) Representative bright-field microscopy images of glomeruli of PAS-stained ${ }^{\text {TiezCre }} D g k e^{\text {fl/fl }}$ mouse kidneys and (B) ${ }^{\text {TiezCre }} D g k e^{\text {fl/fl }}$ Cox2-COE mouse kidneys at 12 weeks of age. The occlusion

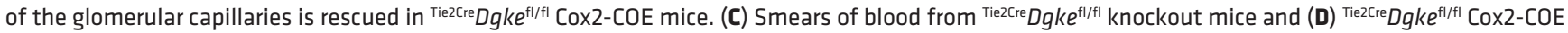
mice at 3 months of age. Schistocytes are visible in Tiezcre $D g k e^{\text {fl/fl }}$ knockout mice (arrowheads) and are absent in ${ }^{\text {Tiezree }} D g k e^{\text {fl/fl }}$ Cox2-COE mice. Scale bars are $50 \mu \mathrm{m}$. (E-H) Serum hemoglobin, circulating PLT, BUN, and urine ACR in ${ }^{\text {TiezCre }} D g k e^{\mathrm{fl} / \mathrm{fl}}$ and ${ }^{\text {TiezCre }} \mathrm{Dg} \mathrm{e}^{\mathrm{fl} / \mathrm{fl}}$ Cox2-COE mice at 8, 10, and 12 weeks of age. Data are presented as mean \pm SD. ${ }^{*}: P<0.05,{ }^{*}: P<0.01$ by Student's $t$ test. $n=6$ mice per group.

and the initiator of coagulation tissue factor (TF) in vitro (45). We tested the expression of $\mathrm{P}$ selectin and $\mathrm{TF}$ in kidney cortexes of ${ }^{\mathrm{Tie} 2 \mathrm{Cre}} \mathrm{Dgke} \mathrm{fl}^{\mathrm{f} / \mathrm{fl}}$ mice at 3 months of age and of plasma thrombin-antithrombin complexes (TAT) and found that all these parameters were significantly increased, compared with control mice (Figure 6, A-C), confirming in vivo in ${ }^{\text {Tie2Cre }} D g k e^{\mathrm{A} / \mathrm{fl}}$ mice that lack Dgke have activation and prothrombotic state of the endothelium. In line with this observation, we also observed increased endothelial susceptibility to procoagulant inflammatory stimuli, by treating ${ }^{\mathrm{Tie} 2 \mathrm{Cre}} D g k e^{\mathrm{f} / \mathrm{fl}}$ mice and controls at 2 months of age with the synthetic double-strand DNA polyinosinic:polycytidylic acid [poly(I:C)], a potent inducer of interferon- $\alpha$ (46) (Figure 6D). Of note, these results suggest that VEGFA signaling controls the activation and the thrombogenic state of the endothelium indirectly through the regulation of $\mathrm{PGE}_{2}$.

$P G E_{2}$ controls the endothelial expression of CXCR4 in the kidney. Stromal-derived factor 1 (SDF-1, also known as CXCL12) is a soluble chemokine expressed in the stromal component of several organs, and CXCR4 is its cognate receptor expressed on cells of the hematopoietic linage and on endothelial cells. In the kidney, like VEGFA, SDF-1 is expressed in podocytes (47-49). The activation of the SDF-1/ CXCR4 signaling in endothelial cells exerts promigratory and proangiogenic effects, and it is required for the development of the renal vasculature (50). Hypoxia is a strong inducer of VEGFA, SDF-1, and CXCR4 $(51,52)$. In addition, high levels of SDF-1 are known to act in a feed-forward loop to further increase CXCR4 expression in endothelial cells (53). Because endothelial disfunction is expected to result in reduced tissue oxygenation, we tested the expression of hypoxia-induced genes in cortex extracts of ${ }^{\text {Tie2Cre }} D g k e^{\mathrm{f} / \mathrm{fl}}$ and control mice and found that the expression of HIF-1 and HIF-2 was higher in ${ }^{\text {Tie2Cre }} D g k e^{\mathrm{f} / \mathrm{fl}}$ kidney cortexes, compared with controls (Figure 7, A and B), as well as finding higher expression of HIF-1 and HIF-2 transcriptional targets SDF-1 and VEGFA (Figure 7, C and D). However, in spite of the hypoxic milieu of the ${ }^{\mathrm{Tie} 2 \mathrm{Cre}} D g k e^{\mathrm{f} / \mathrm{fl}}$ kidney cortexes, the expression of CXCR4 was surprisingly low, compared with controls (Figure 7E). To confirm that the lower levels of CXCR4 in ${ }^{\mathrm{Tie} 2 \mathrm{Cre}} D g k e^{\mathrm{f} / \mathrm{fl}}$ kidneys were caused by differences in CXCR4 expression in endothelial cells and not in other cell populations, we performed flow cytometry analysis of single-cell suspensions of kidney cortexes of ${ }^{\text {Tie2-Cre }} D g k e^{\mathrm{f} / / \mathrm{fl}}$ and control mice double-stained with antibodies against CXCR4 and against the endothelial cell marker VE-Cadherin. Consistent with other reports (54), VE-Cadherin ${ }^{+}$endothelial cells represented about 3\%-4\% of the cells in our samples (Supplemental Figure 6B, Q2 + Q3). This analysis confirmed that, compared with controls, the proportion of CXCR4-expressing endothelial cells was reduced in ${ }^{\text {Tie2-Cre }} \operatorname{Dgke}^{\mathrm{f} / \mathrm{fl}}$ mice 
A
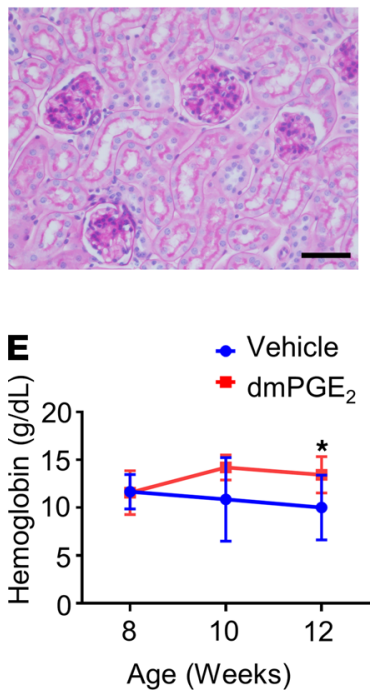

B

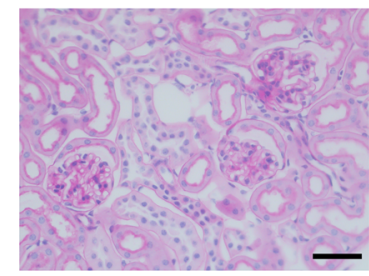

$\mathbf{F}$

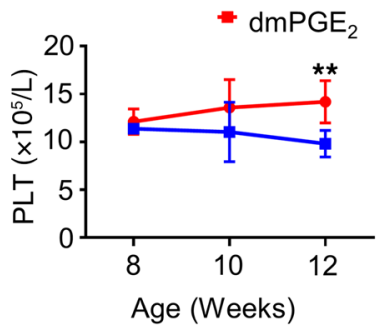

C
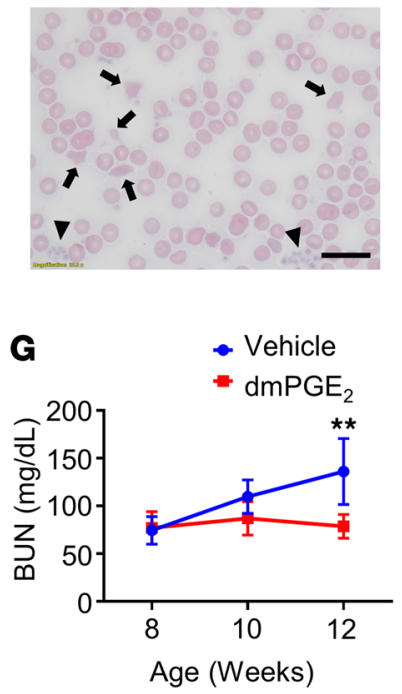

D
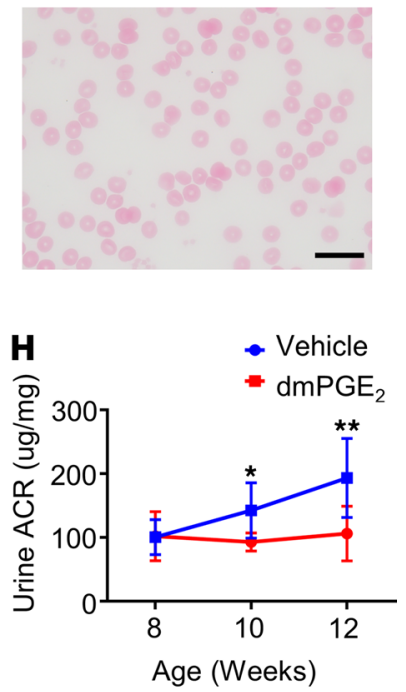

Figure 5. A stable $\mathrm{PGE}_{2}$-analog rescues the phenotype of Tiezcre $D g k e^{f 1 / f 1}$ mice. (A) Representative bright-field microscopy images of glomeruli of PASstained TiezCre $D g k e^{f / f f l}$ mouse kidneys and (B) Tiezcre $D g k e^{f / / f l}$ mouse kidneys after 4 weeks of subcutaneous infusion of the PCE ${ }_{2}$ stable analog dmPGE, The occlusion of the glomerular capillaries is rescued after the dmPGE $\mathrm{E}_{2}$ treatment. Scale bars are $50 \mu \mathrm{m}$. (C) Smears of blood from ${ }^{\text {Tiezcre }} \mathrm{Dgk} \mathrm{e}^{\mathrm{fl} / \mathrm{fl}}$ knockout mice and (D) Tiezcre $D g k e^{\mathrm{fl} / \mathrm{fl}}$ mice after dmPGE treatment at 3 months of age. ${ }^{\text {Tiezcre }} \mathrm{Dg} k e^{\mathrm{fl} / \mathrm{fl}}$ knockout mice show numerous schistocytes (arrows) and circulating reticulocytes (arrowheads). Scale bars are $50 \mu \mathrm{m}$. (E-H) Serum hemoglobin, circulating PLT, BUN, and urine ACR in

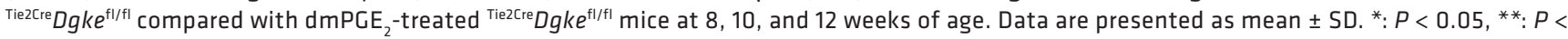
0.01 by Student's $t$ test. $n=6$ mice per group.

(Figure 7, F and G; and Supplemental Figure 6, A and C). Of note, because CXCR4 expression can be induced by $\mathrm{PGE}_{2}$ through the activation of adenylate cyclase (55-57), we tested the expression of CXCR4 in DGKE-knockdown HUVECs and HMECs by Q-PCR and found that CXCR4 expression was strongly downregulated in these cells, but it was rescued to normal levels after supplementation with PGE $_{2}$ (Figure $7 \mathrm{H}$ and Supplemental Figure $2 \mathrm{C}$ ). Remarkably, treatment with $\mathrm{dmPGE}_{2}$ increased the number of endothelial cells expressing CXCR4 in kidney cortexes of ${ }^{\text {Tie2-Cre }} D g k e^{\mathrm{f} / \mathrm{fl}}$ mice (Figure 7, I and J). These results show that in the kidneys, $\mathrm{PGE}_{2}$ controls the endothelial expression of CXCR4 and thus the activity of the SDF-1/CXCR4 signaling axis.

$P G E_{2}$ suppresses the expression of MMP-2 in ${ }^{\mathrm{Tie} 2 \mathrm{Cr}} D g k e^{\mathrm{f} / \mathrm{fl}}$ mouse kidneys. DGKE nephropathy is invariably associated with proteinuria $(3,25,58)$. Proteinuria was also present in $100 \%$ of endothelial specific ${ }^{\text {Tie2-Cre }} D g k e^{\mathrm{f} / \mathrm{fl}}$ knockout mice at a later time compared with the appearance of the hematologic signs, which suggests that the filtration barrier is secondarily affected by primary endothelial abnormalities. To gain insights into the pathogenesis of the proteinuria in the ${ }^{\text {Tie2-Cre }} D g k e^{\mathrm{f} / \mathrm{fl}}$ knockout mice, we performed transmission electron microscopy (TEM) of renal cortexes of these mice at 3 months of age, when albuminuria is detectable. ${ }_{\text {Tie2-Cre }} D g k e^{\mathrm{f} / \mathrm{fl}}$ knockout mouse glomeruli showed moderate podocyte foot process effacement, subendothelial widening, and, interestingly, segmental thickening of the glomerular basement membrane (GBM) (Figure 8, $\mathrm{A}$ and $\mathrm{B})$, but all these abnormalities were not detectable in $\mathrm{dmPGE}_{2}$-treated ${ }^{\mathrm{Tie} 2 \mathrm{Cre}} \mathrm{Dgke} \mathrm{e}^{\mathrm{t} / \mathrm{fl}}$ mice, in concordance with the normalized albuminuria (Figure 8, C and D, and Figure 5F). For this reason, we investigated if the proteinuria in ${ }^{\text {Tie2-Cre }} D g k e^{\mathrm{f} / \mathrm{ll}}$ mice could result from abnormalities of the GBM induced by the activated endothelium (59). In the bone marrow niche, SDF-1 and CXCR4 are essential regulators of the homing of hematopoietic progenitor cells (HPCs) (60-63), and inhibition of CXCR4 is used to force HPCs to egress from their niche into the bloodstream, when large numbers of HPCs need to be harvested for bone marrow transplantation (64). The mobilizing effect of CXCR4 inhibitors and other hematopoietic cell mobilizers is mediated by the induction of several proteinases, including MMPs (65). Considering that the expression of CXCR4 in endothelial cells can be induced by PGE 2 (55-57), we tested by Q-PCR if impaired endothelial induction of $\mathrm{PGE}_{2}$ in the absence of $D g k e$ would be indirectly associated with increased expression of MMP-2 and MMP-9, the MMPs most abundantly expressed in the glomeruli (66), compared with controls. We found that the expression of MMP-2 was increased in DGKE-knockdown HUVECs and HMECs and normalized by supplementing the culture medium with $\mathrm{PGE}_{2}$ (Figure $8 \mathrm{E}$ and Supplemental Figure 2D). 
A

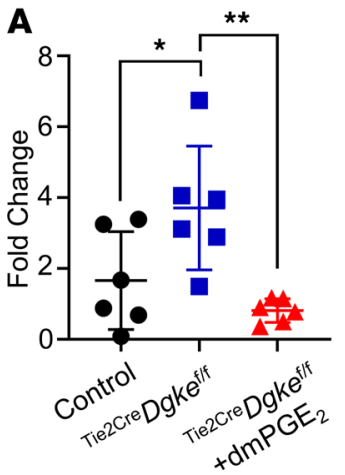

B

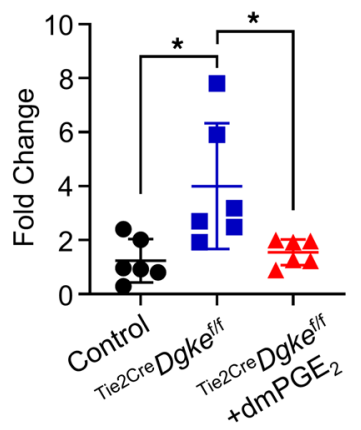

C

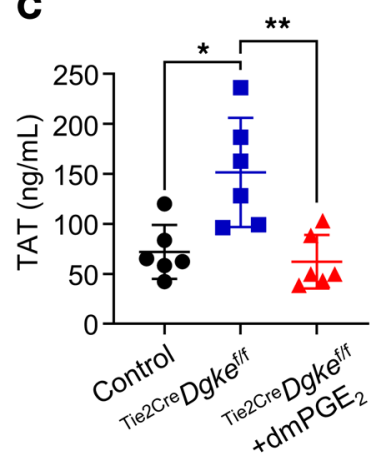

D

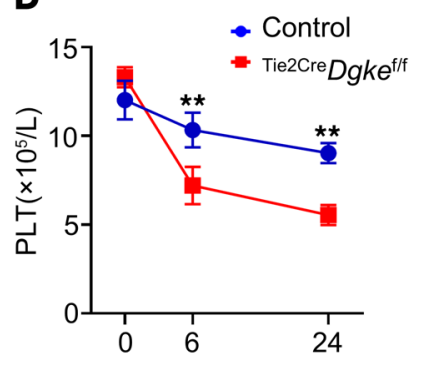

Hours After Poly(I:C) Injection

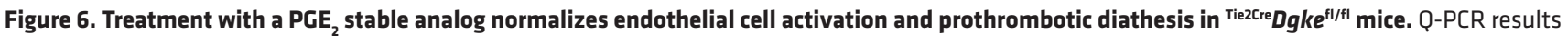
showing increased (A) P selectin and (B) TF expression in kidney cortexes of Tiezre $D g k e^{\text {fl/fl }}$ mice compared with controls that were within normal range in Tiezcre $D g k e^{f l / f l}$ littermates after 4 weeks of subcutaneous infusion of $\mathrm{dmPGE}_{2}$. (C) Thrombin-antithrombin complex (TAT) levels in plasma showing increased

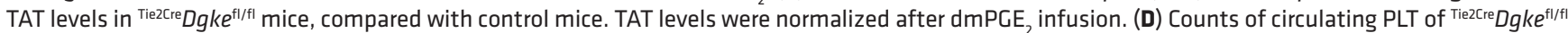
mice and controls at 0,6 , and 24 hours after poly(l:C) injection. Data are presented as mean \pm SD. ${ }^{*}: P<0.05$, ${ }^{* *}: P<0.01$ by 1 -way ANOVA in A-C, where each data point represents 1 mouse, and by Student's $t$ test in $\mathbf{D} ; n=6$ mice per group.

MMP-2, but not MMP-9, expression was also higher in cortex extracts of ${ }^{\text {Tie2-Cre }} D g k e^{\mathrm{f} / \mathrm{fl}}$ mice in vivo, but not in kidneys of mice 28 days after they were injured by folic acid injection (67) (Supplemental Figure 7). We further confirmed by gel zymography analysis of cortex lysates that the total MMP-2 protein levels and its proteolytic activity were increased in ${ }^{\text {Tie2-Cre }} D g k e^{\mathrm{f} / \mathrm{fl}}$ mice but not in ${ }^{\text {Tie2-Cre }} D g k e^{\mathrm{f} / \mathrm{fl}}$ mice treated with $\mathrm{dmPGE}_{2}$ (Figure $8 \mathrm{~F}$ ). These results indicate that MMP-2 activity is high in endothelial specific ${ }^{\text {Tie2-Cre }} D g k e^{\mathrm{fl} / \mathrm{fl}}$ mouse kidneys and suggest that chronic impairment of the VEGF signaling in these mice may favor the remodeling of the GBM, maybe by controlling PGE $\mathrm{P}_{2}$ and CXCR4-dependent MMP-2 expression. Further studies that are beyond the scope of this work will be needed to further explore this possibility.

\section{Discussion}

In this work we have investigated the molecular mechanisms by which biallelic loss-of-function mutations in DGKE cause a form of TMA and aHUS that it is not associated with defects of the alternative complement pathway $(2,3)$. By generating a potentially novel conditional knockout mouse for the gene $D g k e$, we demonstrated that endothelial specific deletion of Dgke is sufficient to phenocopy in full the human disease, which is remarkably similar to the disease reported in subjects treated with bevacizumab (2), highlighting the centrality of the endothelium in the DGKE disorder and suggesting that podocytes, which also express $D G K E$, are only secondarily affected. Mechanistically, we found that the shortage of PIP2, the intracellular substrate of PI3K, is the primary biochemical defect that causes the DGKE disease resulting in defective PIP3-dependent Akt activation in endothelial cells downstream of VEGFR2, as demonstrated in vitro and in vivo by the experiments in which a PTEN inhibitor was administered to ${ }^{\text {Tie2-Cre }} D g k e^{\mathrm{f} / \mathrm{fl}}$ mice. Thus, our data point at a central role of a defect of the Akt signaling in the endothelium of ${ }^{\text {Tiez-Cre }} D g k e^{\mathrm{f} / \mathrm{fl}}$ mice and speak against a potential role of increased PKC activity in determining the DGKE phenotype, although this possibility cannot be completely excluded. Consistent with our in vivo data is also the conclusion, recently reported in an in vitro study, that by causing shortage of PIP2 (the common cellular substrate of both PI3K and PLC) loss of Dgke does not affect DAG signaling (8).

A somewhat unexpected connection that emerges from our studies is the relation between endothelial VEGF and Cox $2 / \mathrm{PGE}_{2}$ signaling in endothelial cells. Although we previously reported the defect of Cox2 expression and $\mathrm{PGE}_{2}$ production in the constitutive $D g k e$-knockout mice, it was not clear how these abnormalities would be connected with the observed phenotype and if their correction would have been able to rescue the disease in $D g k e$-defective mice. The in vitro and in vivo experiments that we have reported here show that Dgke is required in endothelial cells to induce the expression of Cox 2 and the production of $\mathrm{PGE}_{2}$ downstream of VEGFR2 and to promote the maintenance of the kidney microvasculature. This conclusion is supported by the ability of the endothelial overexpression of Cox 2 and of the subcutaneous infusion of $\mathrm{dmPGE}_{2}$ to rescue the hematologic and renal manifestations observed in ${ }^{\text {Tie2-Cre }} D g k e^{\mathrm{f} / \mathrm{fl}}$ mice. To this point, it is worthwhile to notice that treatment with $\mathrm{dmPGE}_{2}$ both rescued capillary occlusion and renal function, which could be explained by a vasodilator effect of $\mathrm{PGE}_{2}$ on the glomerular microvasculature, 
A

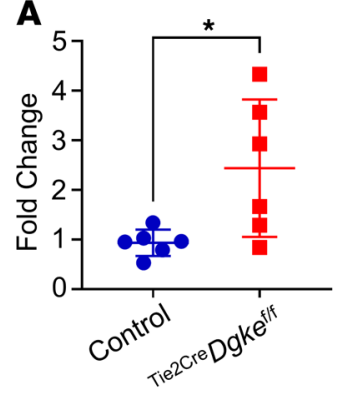

B

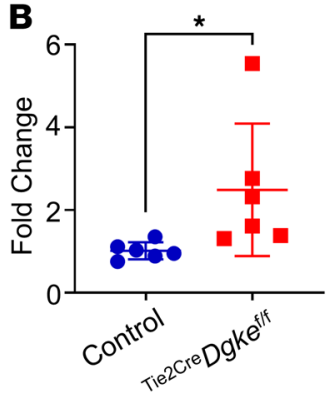

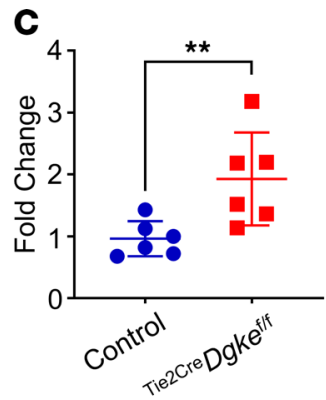
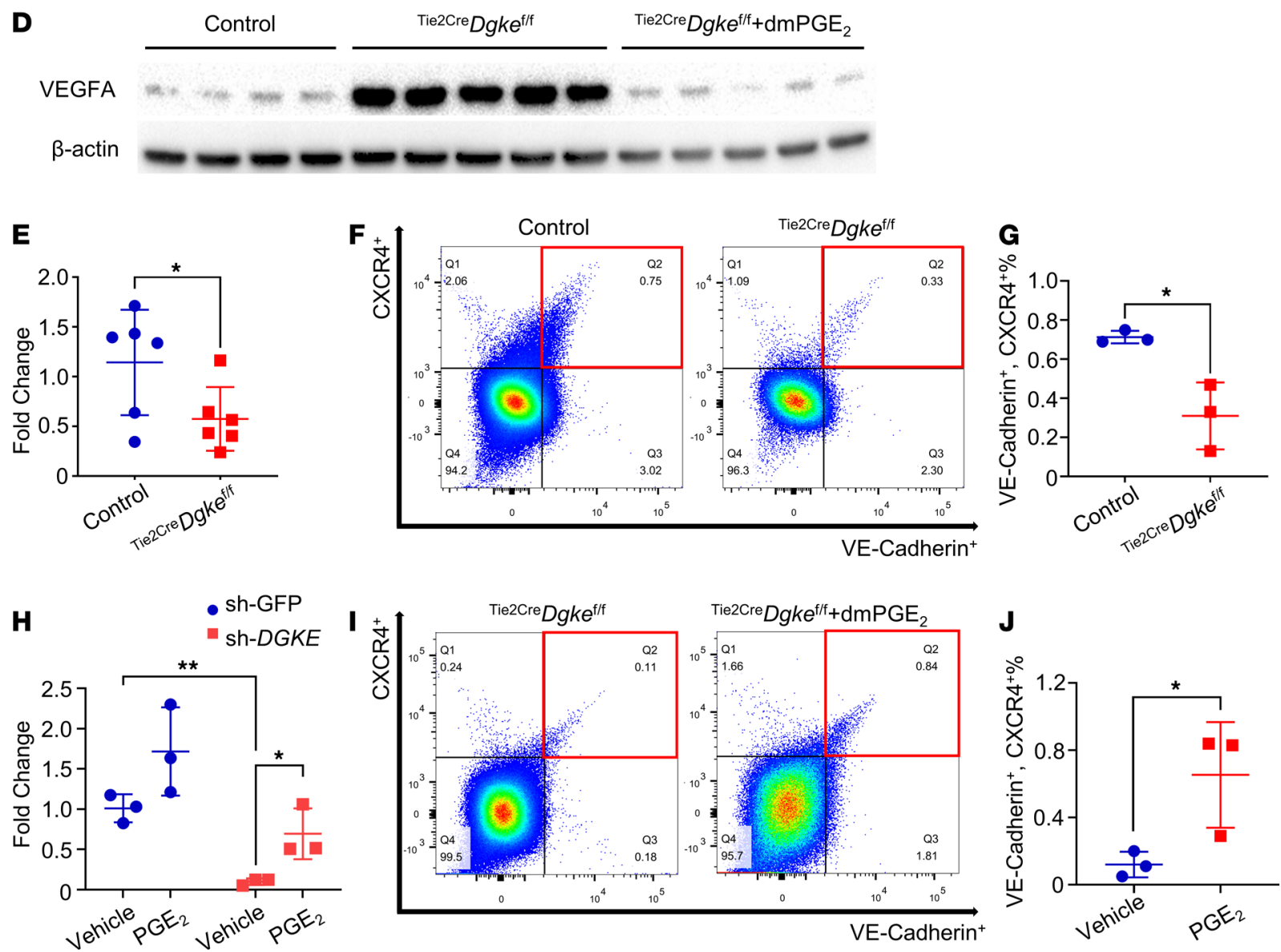

Figure 7. PGE, controls the endothelial expression of CXCR4 in the kidney. Q-PCR showing increased (A) Hif1a, (B) Hif2a, and (C) Sdf-1 expression in kidney cortexes of Tie2Cre $D g k e^{f l / f l}$ mice compared with controls. (D) Western blot showing increased protein levels of VEGFA in kidney cortexes of ${ }^{\text {TiezCre }} D g k e^{f l / f l}$ mice

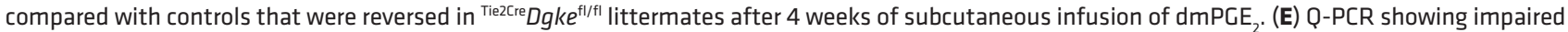
CXCR4 expression in kidney cortexes of Tiezre $D g k e^{\mathrm{f} / \mathrm{fl}}$ mice compared with controls. (F) Representative flow cytometry plots and (C) statistical analysis of 3 independent flow cytometry experiments demonstrating decreased CXCR4 ${ }^{+}$endothelial cells (CXCR4 ${ }^{+}$, VE-Cadherin+) in kidney cortexes of ${ }^{\text {Tiezcre }} D g k e^{f / / f l}$ mice

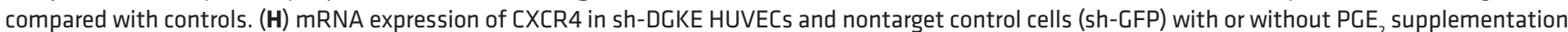
showing that CXCR4 expression is rescued by dmPGE . (I) Representative flow cytometry plots and (J) statistical analysis of 3 independent flow cytometry experiments showing decreased CXCR4 $4^{+}$endothelial cells (CXCR4 ${ }^{+}$, VE-Cadherin ${ }^{+}$) in kidney cortexes from ${ }^{\text {Tiezcre }} D g k e^{\mathrm{fl} / \mathrm{fl}}$ mice. CXCR4 $^{+}$, VE-Cadherin ${ }^{+}$cells increased after 4 weeks' infusion of dmPGE in $^{\text {Tiezcre }} D g k e^{\text {fl/fl }}$ littermates. Data are from 3 independent experiments and are presented as mean $\pm \mathrm{SD}$. ${ }^{* *}: P<$ 0.01 , $^{*} P<0.05$ by 1-way ANOVA in $\mathbf{H}$ and by Student's $t$ test in $\mathbf{A}-\mathbf{C}, \mathbf{E}, \mathbf{G}$, and $\mathbf{J} ; n=3-6$ per group. Each data point represents 1 experiment or 1 mouse.

and avoided the activation of the endothelial cells and the expression of prothrombotic molecules, suggesting that the antiactivating effect of VEGFA on endothelial cells is exerted through the production of $\mathrm{PGE}_{2}$.

Finally, the studies that we have presented here imply an unanticipated interaction between VEGF and SDF-1/CXCR4 signaling in the kidney. A hypothetical general model that could be drawn from our observations would suggest the existence of a balance between proangiogenic VEGF-PGE 2 -mediated signaling that would cause, among other responses, the GBM remodeling by activating MMPs and negative 
A

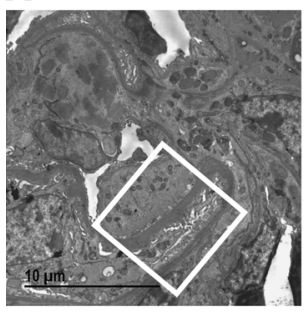

B

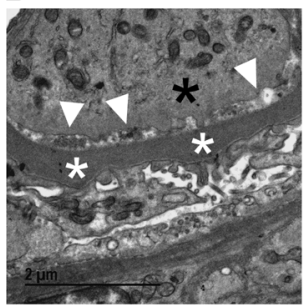

C

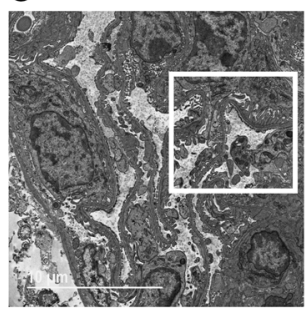

D

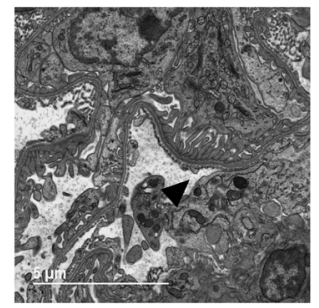

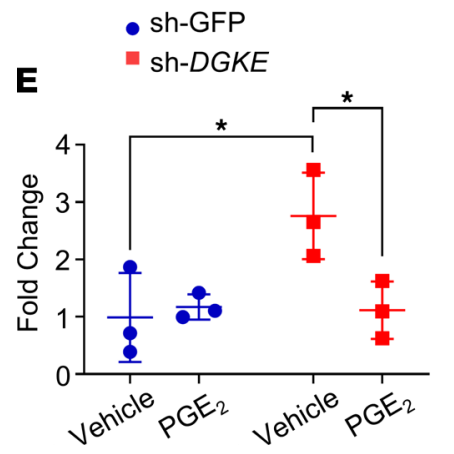

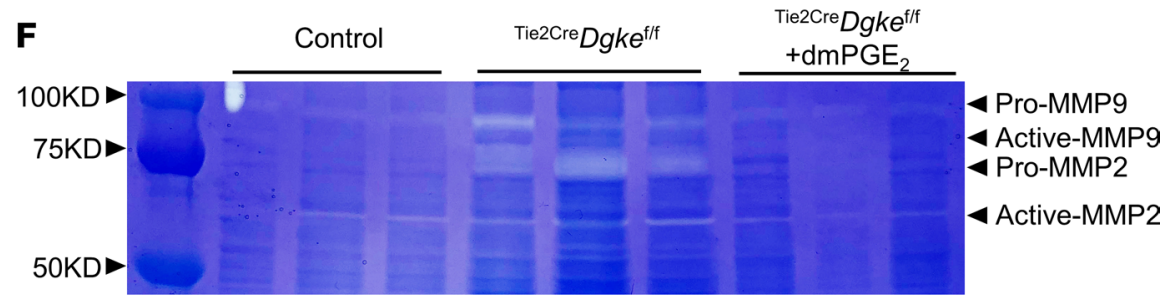

Figure 8. PGE ${ }_{2}$ suppresses the expression of MMP-2 in Tie2cre Dgke ${ }^{\text {fl/fi }}$ mouse kidneys. (A and B) Representative TEM images of a glomerulus of a 3-monthold ${ }^{\text {Tiez } r \text { re }} D g k^{\mathrm{fl} / f \mathrm{fl}}$ mouse. (B) Higher magnification of the inset in $\mathbf{A}$. White arrowheads point at subendothelial widening at the base of a swollen endothelial cell (black asterisk). White asterisks denote thickened GBM; black arrows, effaced foot processes. Scale bars: $10 \mu \mathrm{m}$ (A), $2 \mu \mathrm{m}$ (B). (C and D) Representative TEM image of a glomerulus of a 3-month-old Tiezcre $D g k e^{\text {fl/fl }}$ mouse after 4 weeks' subcutaneous infusion with dmPGE ${ }_{2}$. (D) Higher magnification of the inset in C. Black arrow points to normal foot processes; black arrowhead, healthy fenestrated endothelium and normal GBM. Scale bars: $10 \mu \mathrm{m}$ (C), $5 \mu \mathrm{m}$ (D). (E) Q-PCR showing MMP-2 expression in sh-DGKE-knockdown HUVECs and nontarget control cells (sh-GFP) before and after supplementation of the culture medium with $\mathrm{PCE}_{2}$. Data are from 3 independent experiments and are presented as mean $\pm \mathrm{SD}$. *: $P<0.05$ by 1 -way ANOVA, $n=3$ per group. Each data point represents 1 experiment. (F) Gelatin-polyacrylamide gel showing the zymographic analysis of kidney cortex lysates of Tie2cre $D g k e^{\mathrm{fl} / \mathrm{fl}}$ littermates of the same age after 4 weeks of dmPGE 2 administration. Both pro-MMP-2 $(72 \mathrm{kDa})$ and active MMP-2 $(\sim 60 \mathrm{kDa})$ are present in the ${ }^{\text {Tiezcre }} \mathrm{Dgke} e^{\mathrm{fl} / \mathrm{fl}}$ mouse kidney, compared with controls, but disappear after treatment with $\mathrm{dmPGE}_{2}$.

feedback sustained by the counteracting SDF-1/CXCR4 signaling triggered by $\mathrm{PGE}_{2}$. According to such a model, the lack of Dgke and $\mathrm{PGE}_{2}$ would impair CXCR4-dependent suppression of MMP-2 in endothelial cells, and it would eventually result in glomerular filtration barrier damage (Graphical abstract). Although further experimental evidence has to be produced to better define the interplay between $\mathrm{PGE}_{2}$ and CXCR4 expression in vivo, our results reveal the existence of complex autocrine signaling events downstream of VEGFR2 that could conceivably be manipulated to modify the clinical course of aHUS and of other diseases characterized by endothelial activation or abnormal angiogenesis.

\section{Methods}

Animal studies. All mice were on a C57BL/6J background. The Cox2-COE mice were provided by Harvey R. Herschman, University of California, Los Angeles (Los Angeles, California, USA), and were previously described (38).

Generation of Dgke conditional knockout mice. These mice were generated by injecting ES cells with a knockout first construct, from the KOMP (https://www.komp.org) (30). The knockout first allele is initially a nonexpressive form, but it can be converted to a conditional allele, which carries 2 LoxP sites flanking exon 2 of $D g k e$, via Flp recombination. We confirmed by genomic sequencing that this exon was deleted after Cre recombination. Dgke-floxed mice are viable and fertile, and they produce progeny at the expected Mendelian ratio. Tie2-Cre mice were purchased from The Jackson Laboratory (stock 008863) and were previously described (68). CMV-Cre mice were purchased from The Jackson Laboratory (stock 006054). WT mice bred from the colony were used as controls, unless otherwise specified.

Endothelial cell culture. HUVECs and human dermal microvascular endothelial cells (HMEC-1) were obtained from the American Type Culture Collection. Monolayers were cultured in medium 199 (Gibco, Thermo Fisher Scientific) supplemented with 20\% heat-inactivated FBS (Gibco, Thermo Fisher Scientific), 
endothelial cell growth supplement $(0.03 \mathrm{mg} / \mathrm{mL})$, heparin $(0.1 \mathrm{mg} / \mathrm{mL})$, penicillin $(100 \mathrm{IU} / \mathrm{mL})$, and streptomycin $(100 \mu \mathrm{g} / \mathrm{mL})$. HMEC-1 monolayers were cultured in MCDB131 (Gibco, Thermo Fisher Scientific) supplemented with 15\% heat-inactivated FBS (Gibco, Thermo Fisher Scientific), endothelial cell growth supplement $(20 \mu \mathrm{g} / \mathrm{mL})$, hydrocortisone $(1 \mu \mathrm{g} / \mathrm{mL})$, and glutamine $(10 \mathrm{mM})$ in a humidified incubator at $37^{\circ} \mathrm{C}$ and $5 \% \mathrm{CO}_{2} / 95 \%$ air.

Lentiviral infection and shRNA-mediated gene silencing. DGKE and GFP-targeting shRNA lentiviral constructs were purchased from Thermo Fisher Scientific. The experiments were performed as previously described (69).

In vitro stimulation of HUVECs and HMECs with VEGF, with and without phosphoinositides. Human VEGF recombinant protein was purchased from Gibco (Thermo Fisher Scientific). For VEGF treatment experiments, endothelial cells were first starved for 6 hours, then incubated with $80 \mathrm{ng} / \mathrm{mL}$ VEGF or vehicle control (PBS) for 15 minutes before being used for Western blot or intracellular calcium measurements. For phosphoinositide rescue experiments, cells were first starved for 6 hours. PIP2 or PIP3 (Echelon) was mixed with the carrier histone $\mathrm{H} 1$ (Echelon) at a 1:1 ratio. After a brief water bath sonication for 15 seconds, the mixture was incubated at room temperature for 15 minutes. Then it was added to the cell culture for 1 hour at the final concentration of $2.5 \mu \mathrm{M}$. Cells were then supplemented with VEGF or vehicle (PBS) as described above.

Western blots. Tissues were lysed in lysis buffer (150 mM NaCl, $10 \mathrm{mM}$ EDTA, $10 \mathrm{mM}$ Tris pH 7.4, 1\% Triton X-100, DTT, and 25 mM N-Ethylmaleimide) with protease inhibitor cocktail (Roche) and phosphatase inhibitor cocktail (Roche). Protein concentration was calculated by NanoDrop (Thermo Fisher Scientific). Proteins were separated with NuPAGE 4\%-12\% Bis-Tris Gel (Invitrogen, Thermo Fisher Scientific) at $160 \mathrm{~V}$, then transferred to a nitrocellulose membrane for 90 minutes at $100 \mathrm{~V}$ at $4^{\circ} \mathrm{C}$. The membrane was blocked for 120 minutes in TBS-Tween with 5\% BSA at room temperature and incubated with antibodies against anti-pAkt (threonine 308) (Cell Signaling Technologies, 4056), anti-p-Akt (serine 473) (Cell Signaling Technologies, 9271), anti-Akt (Cell Signaling Technologies, 9272), anti-COX2 (Cell Signaling Technologies, 12282), and anti- $\beta$-actin (Cell Signaling Technologies, 8457) overnight at a dilution of 1:1000. Secondary rabbit antibody (Cell Signaling Technologies, 7074) and secondary mouse antibody (Cell Signaling Technologies, 7076) were incubated for 80 minutes at a concentration of 1:10,000 at room temperature. For exposure, Western ECL Substrate (Bio-Rad) was used. Imaging was done with ChemiDoc XRS+ System with Image Lab Software (Bio-Rad). Western blot densitometry was performed using ImageJ Version 1.53g, 2020 (NIH).

Measurement of intracellular calcium. VEGF-stimulated intracellular calcium release was measured in HUVECs. Briefly, cells were washed in PBS and subsequently loaded with $4 \mu \mathrm{M}$ Fura-2 AM (Thermo Fisher Scientific) in Tyrode's solution ( $140 \mathrm{mM} \mathrm{NaCl}, 5 \mathrm{mM} \mathrm{KCl}, 1.2 \mathrm{mM} \mathrm{CaCl}_{2}, 1 \mathrm{mM} \mathrm{MgCl}, 0.33 \mathrm{mM} \mathrm{NaH}_{2} \mathrm{PO}_{4}$, $5.5 \mathrm{mM}$ glucose, $10 \mathrm{mM} \mathrm{HEPES}$ at $\mathrm{pH} 7.4$ with $\mathrm{NaOH}$ ) for 45 minutes at $37^{\circ} \mathrm{C}$. The cells were then washed in PBS, seeded onto poly-L-lysine-coated glass coverslips, and incubated in complete medium for 1 hour at $37^{\circ} \mathrm{C}$. After incubation, coverslips were transferred to $\mathrm{Ca}^{2+}$-free Tyrode's solution, and the fluorescence ratio of the cells was measured at $340 \mathrm{~nm}$ and $380 \mathrm{~nm}$ at $37^{\circ} \mathrm{C}$ using MetaFluor Imaging Software (Molecular Devices), after 40 seconds of baseline recording ( $\triangle \mathrm{R}$ : fluorescence ratio as $340 \mathrm{~nm} / 380 \mathrm{~nm}$ ).

Quantitative RT-PCR. Total RNA was extracted using the RNeasy Mini Kit (QIAGEN). RNA was reverse-transcribed using the iScript cDNA Synthesis Kit (Bio-Rad). RT-PCR was performed using the CFX Connect Real-Time PCR Detection System (Bio-Rad) and iTaq Universal SYBR Green Supermix (Bio-Rad). All RT-PCR experiments were performed in triplicate. The sequences of PCR primers (IDT) are provided in Supplemental Table 1.

Measurement of BUN, plasma TAT, serum lactate dehydrogenase activity, urine albumin, and urine creatinine. Blood samples were obtained from the retro-orbital plexus. BUN and plasma TAT were measured by ELISA (Abcam), according to the manufacturer's instructions. Twenty-four-hour urine was collected using metabolic cages. Serum lactate dehydrogenase $(\mathrm{LDH})$ activity was tested by $\mathrm{LDH}$ assay kit (MilliporeSigma). Urinary albumin concentration was determined using the Albuwell microalbumin ELISA (Exocell). Urinary creatinine concentration was determined using a P/ACE MDQ Capillary Electrophoresis System and photodiode detector (Beckman Coulter) at the Physiology Core of the O'Brien Center for Kidney Diseases in Dallas (70). ACR was calculated by dividing albumin concentration in milligrams by creatinine concentration in grams.

Measurement of circulating platelets and serum hemoglobin. Mouse blood samples were collected from the retro-orbital plexus using heparinized capillary tubes. After acquisition, samples were immediately processed for platelet counts and hemoglobin measurements on an Advia 120 Hematology system (Siemens Medical Solutions USA Inc.) to avoid clot formation. 
Mouse platelet isolation and in vitro platelet aggregation studies. Mouse platelets were prepared as previously described $(71,72)$. Blood from anesthetized mice was drawn from the retro-orbital plexus and collected in $1.5 \mathrm{~mL}$ polypropylene tubes containing $300 \mu \mathrm{L}$ of enoxaparin $(0.3 \mathrm{mg} / \mathrm{mL}$; Sanofi-Aventis $)$. The blood was centrifuged at $100 \mathrm{~g}$ for 5 minutes, and the platelet-rich plasma (PRP) was collected in a fresh tube. PRP (platelet count; $2 \times 10^{8} / \mathrm{mL}$ ) from WT and endothelial specific knockout DGKE was stirred (178 RCF) at $37^{\circ} \mathrm{C}$ for 2 minutes in a whole blood/optical lumi-aggregometer (Chrono-log, model 700-2) before the addition of agonists (collagen or ADP). Aggregation was measured as percentage change in light transmission, where $100 \%$ refers to transmittance through the blank sample (platelet-poor plasma).

$P G E_{2}$ infusion with subcutaneous osmotic minipumps. Mice were treated with $\mathrm{dmPGE}_{2}$ (Cayman Chemical) and vehicle control through a subcutaneously implanted ALZET 1004 mini-osmotic pump (DURECT). Minipumps were loaded with $100 \mu \mathrm{L}$ of $\mathrm{dmPGE}_{2}$ solution in sterile PBS. A release rate of $0.11 \mu \mathrm{L} / \mathrm{h}$ administered a total amount of $30 \mu \mathrm{g} / \mathrm{kg} \mathrm{dmPGE}{ }_{2}$ daily, a dose that resulted in elevated serum $\mathrm{PGE}_{2}$ without obvious adverse side effects, during the 28 days of the experimental setup.

PTEN inhibitor experiments. Littermate ${ }^{\mathrm{Tie} 2} D g k e^{\mathrm{f} / \mathrm{fl}}$ mice were treated with daily i.p. injection of the PTEN inhibitor VO-OHpic and vehicle control. VO-OHpic (BioVision, 1801-5) was used at $10 \mathrm{mg} / \mathrm{kg} / \mathrm{d}$, administered in a $10 \%$ DMSO solution.

Poly (I:C) injections. Mice were treated with i.p. injections $(1 \mu \mathrm{g} / \mu \mathrm{L}, 200 \mu \mathrm{g} /$ mouse $)$ of a solution of poly(I:C) (MilliporeSigma, P1530). Blood and urine samples were collected at 6 hours, 24 hours, and 7 days after. Platelets, BUN, and ACR were measured as described above.

Flow cytometry. Fresh mouse kidney cortexes were digested in $2 \mathrm{~mL}$ of protease solution $-5 \mathrm{mM}$ $\mathrm{CaCl}_{2}, 10 \mathrm{mg} / \mathrm{mL}$ Bacillus licheniformis protease (Creative Enzymes, NATE0633), and $125 \mathrm{U} / \mathrm{mL}$ DNase I (Roche) in Dulbecco's PBS - on ice for 30 minutes. The lysates were filtered through $70 \mu \mathrm{m}$ cell strainers. Single-cell suspension was collected by rinsing with FACS buffer. Suspensions were lysed with red blood cell lysis buffer (Quality Biological) to remove red blood cells. After washing with $1 \times \mathrm{PBS}, 1 \times 10^{6}$ cells were resuspended in $100 \mu \mathrm{L}$ of FACS buffer and preincubated with anti-mouse CD16/32 antibody (BioLegend, 101302) for blocking prior to labeling with CD144-APC (BioLegend, 138012) and CD184-BV421 (BD Biosciences, 562738) antibodies for 30 minutes at room temperature. Cells were then washed with FACS buffer and analyzed on BD LSR II Violet at the University of Iowa Flow Cytometry Facility. All flow cytometry results were analyzed using FlowJo software (Version 9).

Zymography. Kidney cortical tissues were lysed in lysis buffer $(20 \mathrm{mM}$ Tris- $\mathrm{HCl}, 125 \mathrm{mM} \mathrm{NaCl}$, and $1 \%$ Triton X-100, $\mathrm{pH} 8.5$ ) with protease inhibitor cocktail (Roche). Protein concentration was calculated by NanoDrop (Thermo Fisher Scientific). Samples were separated on Tris-glycine gels with $0.1 \%$ gelatin (Invitrogen, Thermo Fisher Scientific). After electrophoresis, the gels were incubated in $1 \times$ renaturing buffer (Thermo Fisher Scientific) for 2 hours at room temperature, then in $1 \times$ developing buffer (Thermo Fisher Scientific) for 48 hours. Gels were stained with Coomassie Blue R-250 (Bio-Rad) and destained in 20\% ethanol and 10\% acetic acid. MMP-2 activity was detected as clear bands on the blue background.

Statistics. All quantitative data are presented as mean \pm SD. Statistical significance $(P \leq 0.05$ was considered significant) was calculated using the 2-tailed unpaired Student's $t$ test for 2 groups and 1-way ANOVA for multiple-group comparison by Prism 7 (GraphPad Software).

Study approval. All procedures were approved by the Institutional Animal Care and Use Committee of the University of Iowa (protocol 7021976).

\section{Author contributions}

DL performed experiments, analyzed data, prepared figures, and contributed to writing the manuscript. QD, MP, BP, CL, MP, DFD, and MKN performed experiments and analyzed data. HJ, AKC, CS, and $\mathrm{CLH}$ provided reagents. MA designed experiments, analyzed data, prepared figures, and contributed to writing of the manuscript.

\section{Acknowledgments}

The flow cytometry data were obtained at the Flow Cytometry Facility, which is a Carver College of Medicine/Holden Comprehensive Cancer Center core research facility at the University of Iowa. This facility is funded through user fees and the financial support of the Carver College of Medicine, Holden Comprehensive Cancer Center, and Iowa City Veterans Administration Medical Center. Research reported in this publication was supported by the National Center for Research Resources of the NIH under award number 1 
S10 OD016199-01A1. The AKC lab is supported by grants from the NIH (R35HL139926, R01NS109910, U01NS113388) and by the Established Investigator Award 18EIA33900009 from the American Heart Association. We want to thank Harvey R. Herschman, for providing us the Cox2-COE mice.

Address correspondence to: Massimo Attanasio, University of Iowa, CBRB, 3270B, 285 Newton Road, Iowa City, Iowa 52242, USA. Phone: 319.335.9403; Email: massimo-attanasio@uiowa.edu.

1. Noris M, Remuzzi G. Atypical hemolytic-uremic syndrome. N Engl J Med. 2009;361(17):1676-1687.

2. Ozaltin F, et al. DGKE variants cause a glomerular microangiopathy that mimics membranoproliferative GN. J Am Soc Nephrol. 2013;24(3):377-384

3. Lemaire M, et al. Recessive mutations in DGKE cause atypical hemolytic-uremic syndrome. Nat Genet. 2013;45(5):531-536.

4. Tang W, et al. Molecular cloning of a novel human diacylglycerol kinase highly selective for arachidonate-containing substrates. J Biol Chem. 1996;271(17):10237-10241.

5. Milne SB, et al. Dramatic differences in the roles in lipid metabolism of two isoforms of diacylglycerol kinase. Biochemistry. 2008;47(36):9372-9379.

6. Rodriguez de Turco EB, et al. Diacylglycerol kinase epsilon regulates seizure susceptibility and long-term potentiation through arachidonoyl- inositol lipid signaling. Proc Natl Acad Sci U S A. 2001;98(8):4740-4745.

7. Shulga YV, et al. Molecular species of phosphatidylinositol-cycle intermediates in the endoplasmic reticulum and plasma membrane. Biochemistry. 2010;49(2):312-317.

8. So V, et al. Phosphatidylinositol cycle disruption is central to atypical hemolytic-uremic syndrome caused by diacylglycerol kinase epsilon deficiency [preprint]. https://doi.org/10.1101/633867. Published on bioRxiv May 27, 2019.

9. Xu C, et al. Kinetic analysis of receptor-activated phosphoinositide turnover. J Cell Biol. 2003;161(4):779-791.

10. Willars GB, et al. Differential regulation of muscarinic acetylcholine receptor-sensitive polyphosphoinositide pools and consequences for signaling in human neuroblastoma cells. J Biol Chem. 1998;273(9):5037-5046.

11. Creba JA, et al. Rapid breakdown of phosphatidylinositol 4-phosphate and phosphatidylinositol 4,5-bisphosphate in rat hepatocytes stimulated by vasopressin and other Ca2+-mobilizing hormones. Biochem J. 1983;212(3):733-747.

12. Pike LJ, Casey L. Localization and turnover of phosphatidylinositol 4,5-bisphosphate in caveolin-enriched membrane domains. J Biol Chem. 1996;271(43):26453-26456.

13. Koch S, Claesson-Welsh L. Signal transduction by vascular endothelial growth factor receptors. Cold Spring Harb Perspect Med. 2012;2(7):a006502.

14. Koch S, et al. Signal transduction by vascular endothelial growth factor receptors. Biochem. 2011;437(2):169-183.

15. Olsson AK, et al. VEGF receptor signalling - in control of vascular function. Nat Rev Mol Cell Biol. 2006;7(5):359-371.

16. Zachary I. Signaling mechanisms mediating vascular protective actions of vascular endothelial growth factor. Am J Physiol Cell Physiol. 2001;280(6):C1375-C1386.

17. Bartlett CS, et al. Vascular growth factors and glomerular disease. Annu Rev Physiol. 2016;78:437-461.

18. Takahashi T, et al. A single autophosphorylation site on KDR/Flk-1 is essential for VEGF-A-dependent activation of PLC-gamma and DNA synthesis in vascular endothelial cells. EMBO J. 2001;20(11):2768-2778.

19. Cantley LC. The phosphoinositide 3-kinase pathway. Science. 2002;296(5573):1655-1657.

20. Gerber HP, et al. Vascular endothelial growth factor regulates endothelial cell survival through the phosphatidylinositol 3'-kinase/Akt signal transduction pathway. Requirement for Flk-1/KDR activation. J Biol Chem. 1998;273(46):30336-30343.

21. Graupera M, et al. Angiogenesis selectively requires the p110alpha isoform of PI3K to control endothelial cell migration. Nature. 2008;453(7195):662-666

22. Dayanir V, et al. Identification of tyrosine residues in vascular endothelial growth factor receptor-2/FLK-1 involved in activation of phosphatidylinositol 3-kinase and cell proliferation. J Biol Chem. 2001;276(21):17686-17692.

23. Eremina V, et al. Glomerular-specific alterations of VEGF-A expression lead to distinct congenital and acquired renal diseases. J Clin Invest. 2003;111(5):707-716.

24. Sison K, et al. Glomerular structure and function require paracrine, not autocrine, VEGF-VEGFR-2 signaling. J Am Soc Nephrol. 2010;21(10):1691-1701.

25. Eremina V, et al. VEGF inhibition and renal thrombotic microangiopathy. N Engl J Med. 2008;358(11):1129-1136.

26. Akarasereenont PC, et al. The expression of COX-2 in VEGF-treated endothelial cells is mediated through protein tyrosine kinase. Mediators Inflamm. 2002;11(1):17-22.

27. Tamura M, et al. Vascular endothelial growth factor up-regulates cyclooxygenase-2 expression in human endothelial cells. $J$ Clin Endocrinol Metab. 2002;87(7):3504-3507.

28. Wheeler-Jones C, et al. Vascular endothelial growth factor stimulates prostacyclin production and activation of cytosolic phospholipase A2 in endothelial cells via p42/p44 mitogen-activated protein kinase. FEBS Lett. 1997;420(1):28-32.

29. Kage K, et al. Basic fibroblast growth factor induces cyclooxygenase-2 expression in endothelial cells derived from bone. Biochem Biophys Res Commun. 1999;254(1):259-263.

30. Skarnes WC, et al. A conditional knockout resource for the genome-wide study of mouse gene function. Nature. 2011;474(7351):337-342.

31. Osterwalder M, et al. Dual RMCE for efficient re-engineering of mouse mutant alleles. Nat Methods. 2010;7(11):893-895.

32. Schwenk F, et al. A cre-transgenic mouse strain for the ubiquitous deletion of loxP-flanked gene segments including deletion in germ cells. Nucleic Acids Res. 1995;23(24):5080-5081.

33. Moeller MJ, et al. Podocyte-specific expression of cre recombinase in transgenic mice. Genesis. 2003;35(1):39-42.

34. Venneri MA, et al. Identification of proangiogenic TIE2-expressing monocytes (TEMs) in human peripheral blood and cancer Blood. 2007;109(12):5276-5285. 
35. Groszer M, et al. Negative regulation of neural stem/progenitor cell proliferation by the Pten tumor suppressor gene in vivo. Science. 2001;294(5549):2186-2189.

36. Mak LH, et al. Characterisation of the PTEN inhibitor VO-OHpic. J Chem Biol. 2010;3(4):157-163.

37. Zhu J, et al. Loss of diacylglycerol kinase epsilon in mice causes endothelial distress and impairs glomerular Cox-2 and PGE2 production. Am J Physiol Renal Physiol. 2016;310(9):F895-F908.

38. Kamei K, et al. Transgenic mouse for conditional, tissue-specific Cox-2 overexpression. Genesis. 2006;44(4):177-182.

39. Rao R, et al. Prostaglandin E2-EP4 receptor promotes endothelial cell migration via ERK activation and angiogenesis in vivo. J Biol Chem. 2007;282(23):16959-16968.

40. Aoudjit L, et al. Prostaglandin E2 promotes cell survival of glomerular epithelial cells via the EP4 receptor. Am J Physiol Renal Physiol. 2006;290(6):F1534-F1542.

41. Gliki G, et al. Vascular endothelial growth factor-induced prostacyclin production is mediated by a protein kinase C (PKC)-dependent activation of extracellular signal-regulated protein kinases 1 and 2 involving PKC-delta and by mobilization of intracellular Ca2+. Biochem J. 2001;353(pt 3):503-512.

42. Murphy JF, Fitzgerald DJ. Vascular endothelial growth factor induces cyclooxygenase-dependent proliferation of endothelial cells via the VEGF-2 receptor. FASEB J. 2001;15(9):1667-1669.

43. Chang SH, et al. Role of prostaglandin E2-dependent angiogenic switch in cyclooxygenase 2-induced breast cancer progression. Proc Natl Acad Sci U S A. 2004;101(2):591-596.

44. Zhu J, et al. Loss of diacylglycerol kinase epsilon in mice causes endothelial distress and impairs glomerular Cox-2 and PGE(2) production. Am J Physiol Renal Physiol. 2016;310(9):F895-F908.

45. Bruneau S, et al. Loss of DGKe induces endothelial cell activation and death independently of complement activation. Blood. 2015;125(6):1038-1046.

46. Devendra D, Eisenbarth GS. Interferon alpha--a potential link in the pathogenesis of viral-induced type 1 diabetes and autoimmunity. Clin Immunol. 2004;111(3):225-233.

47. Sayyed SG, et al. Podocytes produce homeostatic chemokine stromal cell-derived factor-1/CXCL12, which contributes to glomerulosclerosis, podocyte loss and albuminuria in a mouse model of type 2 diabetes. Diabetologia. 2009;52(11):2445-2454

48. Romoli S, et al. CXCL12 blockade preferentially regenerates lost podocytes in cortical nephrons by targeting an intrinsic podocyte-progenitor feedback mechanism. Kidney Int. 2018;94(6):1111-1126.

49. Ding M, et al. Loss of the tumor suppressor Vhlh leads to upregulation of Cxcr4 and rapidly progressive glomerulonephritis in mice. Nat Med. 2006;12(9):1081-1087.

50. Takabatake Y, et al. The CXCL12 (SDF-1)/CXCR4 axis is essential for the development of renal vasculature. J Am Soc Nephrol. 2009;20(8):1714-1723.

51. Zagzag D, et al. Hypoxia- and vascular endothelial growth factor-induced stromal cell-derived factor-1alpha/CXCR4 expression in glioblastomas: one plausible explanation of Scherer's structures. Am J Pathol. 2008;173(2):545-560.

52. Zagzag D, et al. Stromal cell-derived factor-1alpha and CXCR4 expression in hemangioblastoma and clear cell-renal cell carcinoma: von Hippel-Lindau loss-of-function induces expression of a ligand and its receptor. Cancer Res. 2005;65(14):6178-6188.

53. Salcedo R, et al. Vascular endothelial growth factor and basic fibroblast growth factor induce expression of CXCR4 on human endothelial cells: in vivo neovascularization induced by stromal-derived factor-1alpha. Am J Pathol. 1999;154(4):1125-1135.

54. Park J, et al. Single-cell transcriptomics of the mouse kidney reveals potential cellular targets of kidney disease. Science. 2018;360(6390):758-763.

55. Hoggatt J, et al. Prostaglandin E2 enhances hematopoietic stem cell homing, survival, and proliferation. Blood. 2009;113(22):5444-5455.

56. Hoggatt J, et al. Differential stem- and progenitor-cell trafficking by prostaglandin E2. Nature. 2013;495(7441):365-369.

57. Salcedo R, et al. Angiogenic effects of prostaglandin E2 are mediated by up-regulation of CXCR4 on human microvascular endothelial cells. Blood. 2003;102(6):1966-1977.

58. Brocklebank V, et al. Long-term outcomes and response to treatment in diacylglycerol kinase epsilon nephropathy. Kidney Int. 2020;97(6):1260-1274.

59. Jarad G, et al. Proteinuria precedes podocyte abnormalities inLamb2-/- mice, implicating the glomerular basement membrane as an albumin barrier. J Clin Invest. 2006;116(8):2272-2279.

60. Teicher BA, Fricker SP. CXCL12 (SDF-1)/CXCR4 pathway in cancer. Clin Cancer Res. 2010;16(11):2927-2931.

61. Petit I, et al. The SDF-1-CXCR4 signaling pathway: a molecular hub modulating neo-angiogenesis. Trends Immunol. 2007;28(7):299-307.

62. Jin DK, et al. Cytokine-mediated deployment of SDF-1 induces revascularization through recruitment of CXCR4+ hemangiocytes. Nat Med. 2006;12(5):557-567.

63. Greenbaum A, et al. CXCL12 in early mesenchymal progenitors is required for haematopoietic stem-cell maintenance. Nature. 2013;495(7440):227-230.

64. [No authors listed]. Plerixafor:AMD 3100, AMD3100, JM 3100, SDZ SID 791. Drugs R D. 2007;8(2):113-119.

65. Jujo K, et al. CXCR4 blockade augments bone marrow progenitor cell recruitment to the neovasculature and reduces mortality after myocardial infarction. Proc Natl Acad Sci U S A. 2010;107(24):11008-11013.

66. Sekiuchi M, et al. Expression of matrix metalloproteinases 2 and 9 and tissue inhibitors of matrix metalloproteinases 2 and 1 in the glomeruli of human glomerular diseases: the results of studies using immunofluorescence, in situ hybridization, and immunoelectron microscopy. Clin Exp Nephrol. 2012;16(6):863-874.

67. Jin H, et al. Epithelial innate immunity mediates tubular cell senescence after kidney injury. JCI Insight. 2019;4(2):125490.

68. Kisanuki YY, et al. Tie2-Cre transgenic mice: a new model for endothelial cell-lineage analysis in vivo. Dev Biol. 2001;230(2):230-242.

69. Li B, et al. Increased hedgehog signaling in postnatal kidney results in aberrant activation of nephron developmental programs. Hum Mol Genet. 2011;20(21):4155-4166.

70. Zinellu A, et al. Plasma creatinine and creatine quantification by capillary electrophoresis diode array detector. Anal Biochem. 2005;342(2):186-193. 
71. Nayak MK, et al. Dichloroacetate, an inhibitor of pyruvate dehydrogenase kinases, inhibits platelet aggregation and arterial thrombosis. Blood Adv. 2018;2(15):2029-2038.

72. Nayak MK, et al. Metabolic enzyme pyruvate kinase M2 regulates platelet function and arterial thrombosis. Blood. 2021;137(12):1658-1668. 